\title{
Monotone Data Samples Do Not Always Produce Monotone Fuzzy If- Then Rules: Learning with $A d$ hoc and System Identification Methods
}

\author{
${ }^{1}$ Chin Ying Teh, ${ }^{1 *}$ Kai Meng Tay, ${ }^{2}$ Chee Peng Lim \\ ${ }^{1}$ Department of Electronic Engineering, Faculty of Engineering, Universiti Malaysia Sarawak, Malaysia. \\ ${ }^{2}$ Institute for Intelligent Systems Research and Innovation, Deakin University, Australia \\ *Corresponding author:kmtay@unimas.my,tkaimeng@yahoo.com
}

\begin{abstract}
In this paper, ad hoc and system identification methods are used to generate fuzzy If-Then rules for a zeroorder Takagi-Sugeno-Kang (TSK) Fuzzy Inference System (FIS) using a set of multi-attribute monotone data. Convex and normal trapezoidal fuzzy sets, with a strong fuzzy partition strategy, is employed. Our analysis shows that even with multi-attribute monotone data, non-monotone fuzzy IfThen rules can be produced using an ad hoc method. The same observation can be made, empirically, using a system identification method, e.g., a derivative-based optimization method and the genetic algorithm. This finding is important for modeling a monotone FIS model, as the result shows that even with a "clean" data set pertaining to a monotone system, the generated fuzzy If-Then rules may need to be preprocessed, before being used for FIS modeling. As such, monotone fuzzy rule relabeling is useful. Besides that, a constrained non-linear programming method for FIS modelling is suggested, as a variant of the system identification method.
\end{abstract}

Keywords- Fuzzy If-Then rules, TSK Fuzzy inference system, machine learning, monotonicity, multi-attribute monotone data, monotone fuzzy rule relabeling

\section{INTRODUCTION}

Fuzzy Inference Systems (FISs) with fuzzy If-Then rules have been successfully applied to many real life applications [1-2]. In many applications, fuzzy If-Then rules are obtained from human experts, i.e., knowledge-driven FIS models. Owing to the difficulty to obtain fuzzy rules from human experts, many methods to automatically generate fuzzy If-Then rules from numerical data, i.e., data-driven FIS models, have been proposed. Among the popular methods are system identification [2], gradient descent learning [3-6], fuzzy-neural [7], fuzzy c-means clustering [8], least-square [9-10], and ad hoc methods [4] [11-13].

In this paper, two types of data-driven FIS models for generating monotone fuzzy rules using multi-attribute monotone data are analyzed. They are (i) ad hoc methods [11], i.e., Wang et al. [12-13] (hereafter abbreviated as the Wang-Mendel (WM) method) and Ishibuchi et al. [4] (hereafter abbreviated as the Ishibuchi method); and (ii) system identification methods, i.e., derivative-based optimization [14-17] and Genetic Algorithm (GA)-based methods [18]. We adopt the classification terminologies in [19], in which both WM [12-13] and Ishibuchi [4] [11] methods are known as "ad hoc methods". Besides that, we refer to [2] for the general framework of a system identification method to learn an FIS model.

Even though data-driven FIS models are popular, research on monotone data-driven FIS models is new. The importance of the monotonicity property in FIS modeling has been highlighted in a number of recent publications [2027], and a number of studies to preserve the monotonicity property have been proposed. These include the development of mathematical conditions as a set of governing equations [20-22], application of the developed mathematical conditions to real-world problems [23], as well as extension and synthesis of the mathematical conditions with some advanced FIS modeling techniques [23-25]. Our earlier studies on construction of a monotone FIS model from data have been reported in [28-29].

In this paper, monotone data sets (as in Definition 6) are considered. The hypothesis is to ascertain whether ad hoc and system identification methods could always produce monotone fuzzy rules with multi-attribute monotone data sets. Convex and normal trapezoidal fuzzy sets are used as the fuzzy membership functions (Definition 2). Besides that, a strong fuzzy partition strategy [26] is used (Definition 3). Our analytical results suggest that fuzzy IfThen rules generated using ad hoc methods do not always satisfy the monotonicity property, even though the multiattribute data are monotone. A simulated example is used for illustration. The same observation can be made, empirically, using system identification methods, in which a derivative-based optimization method and the GA are used to search for a (near) optimal solution. This is important, as we show that a local (potentially global) optimal solution from a system identification method may not be feasible (in this paper, we define a set of monotone fuzzy rules as a feasible solution).

This finding is useful in practical applications, because given a "clean" data set (free from noise, i.e., monotone data), the generated fuzzy If-Then rules from a monotone system may need to be pre-processed (e.g., re-labeled) in order to satisfy the monotonicity property pertaining to FIS modelling. Our monotone fuzzy rule relabeling technique [30] coupled with ad hoc methods is a solution for this problem. In this paper, fuzzy rules generated from ad hoc methods are re-labeled based on the following criteria: (i) fulfilling the monotonicity property (Definition 4.3); and (ii) having the minimum loss measure. Note that other sets of criteria for fuzzy rule relabeling could also be applied, and this constitutes a good direction for further research. In addition to the system identification methods, an alternative system identification formulation, which includes monotone fuzzy rules as constraints, is also presented. The empirical results show that including monotone fuzzy rules as constraints provides more reasonable results. 